\title{
DESTETE PRECOZ Y DESEMPEÑO REPRODUCTIVO EN VACAS TRATADAS CON PROGESTERONA INTRAVAGINAL
}

\author{
EARLY WEANING IN BEEF COWS TREATED WITH INTRAVAGINAL PROGESTERONE \\ AND THEIR REPRODUCTIVE PERFORMANCE
}

\author{
Vittone, J.S. ${ }^{1 *}$, Aller, J.F. ${ }^{2}$, Otero, G. ${ }^{1}$, Scena, C. ${ }^{3}$, Alberio, R.H. ${ }^{2}$ y Cano, A. ${ }^{2}$ \\ ${ }^{1}$ Instituto Nacional de Tecnología Agropecuaria. EEA Concepción del Uruguay. Entre Ríos. Argentina. \\ *jvittone@concepcion.inta.gov.ar \\ 2Instituto Nacional de Tecnología Agropecuaria. EEA Balcarce. Balcarce. Argentina. \\ ${ }^{3}$ Alimental S.A. Venado Tuerto Santa Fe. Argentina.
}

\section{Palabras claVe adicionales}

Vacas de cría. Inseminación artificial a tiempo fijo.

\section{RESUMEN}

El objetivo del presente trabajo fue determinar el efecto del momento del destete precoz (DP) en vacas con cría tratadas con progesterona intravaginal sobre las características de la ovulación y la eficiencia reproductiva. Se realizaron 2 experimentos con animales de raza Hereford y Polled-Hereford. Exp.1: 38 vacas de baja condición corporal $(3,3 \pm 0,8 ;$ media $\pm D E$; escala de 1 a 9$)$ con $55,7 \pm 8,8$ días posparto y Exp. 2: 35 vacas de alta condición corporal $(5,4 \pm 0,9)$ con $57,5 \pm 15,9$ días posparto. El día 0 todos los animales recibieron un dispositivo intravaginal (DIV) impregnado con $0,5 \mathrm{~g}$ de progesterona más $2 \mathrm{mg}$ de benzoato de estradiol (BE; im). El día 8 se retiró el DIV, se aplicó $500 \mu \mathrm{g}$ de cloprostenol sódico (im) y 24 horas después se aplicó $1 \mathrm{mg}$ de BE. Los animales fueron distribuidos aleatoriamente a 4 tratamientos: T1): DP diez días antes de colocar el DIV; T2): DP el día de colocación del DIV; T3): DP el día del retiro del DIV y T4): Control con cría al pie. Todas las vacas fueron inseminadas a tiempo fijo a las 56 $\mathrm{h}$ del retiro de los DIV. En ambos experimentos se evaluó mediante ultrasonografía la dinámica folicular, el momento y distribución de las ovulaciones y la preñez. Se determinó la concentración plasmática de progesterona para evaluar la funcionalidad y la vida media del cuerpo lúteo generado por la ovulación inducida en los vientres que no resultaron preñados. En vacas de baja condición corporal (Exp.1), el diámetro del folículo dominante al retiro del DIV en el tratamiento T2

\section{AdDitionAL KEYWORDS}

Beef cows. Fixed-time artificial insemination.

$(11,2 \pm 2,5 \mathrm{~mm})$ fue mayor $(p<0,05)$ que en el tratamiento T3 $(7,3 \pm 2,0 \mathrm{~mm})$, no registrándose diferencias con los tratamientos T1 y T4 $(10,3 \pm 2,4$ y $8,2 \pm 2,8 \mathrm{~mm}$, respectivamente). La tasa de ovulación fue mayor $(p<0,05)$ en los tratamientos $\mathrm{T} 1$ y $\mathrm{T} 2(77,8 \%$ y $88,9 \%$, respectivamente) que en los tratamientos T3 y T4 (40\% en ambos casos). La tasa de preñez en el tratamiento T2 $(77,8 \%)$ fue superior $(p<0,05)$ al resto de los tratamientos evaluados ( $\mathrm{T} 1=55,6 \%, \mathrm{~T} 3=30 \%$ y $\mathrm{T} 4=20 \%$ ). En vacas de alta condición corporal (Exp. 2) no se observaron diferencias $(p>0,05)$ entre tratamientos en ninguno de los parámetros evaluados. La vida media del cuerpo lúteo de las vacas no gestantes fue normal, independientemente del tratamiento aplicado y de la condición corporal de las mismas. Este estudio permitió determinar que, en vacas de baja condición corporal, el DP realizado 10 días antes o al inicio de un tratamiento con progesterona/estrógenos produjo un mayor tamaño del folículo dominante (preovulatorio) y una mayor tasa de ovulación. Sin embargo, la tasa de preñez sólo fue mejorada cuando el destete se realizó al inicio del tratamiento hormonal en las vacas de baja condición corporal asignadas al Exp.1.

\section{SUMMARY}

The objective of the present study was to determine the effect of early weaning time on the 


\section{VITTONE, ALLER, OTERO, SCENA, ALBERIO Y CANO}

ovulation characteristics and the reproductive efficiency of treated beef cows with an intravaginal progesterone device (DIV). Two experiments were performed utilizing Hereford and Polled Hereford cows. Exp. 1: 38 low body condition score cows (BCS; $3.3 \pm 0.8$, mean $\pm S D$, range from 1 to 9 ) with $57.5 \pm 8.8$ days postpartum and Exp. $2: 35$ high BCS $(5.4 \pm 0.9)$ cows with $57.5 \pm 15.9$ days postpartum. On day 0 , all cows received an DIV containing 0.5 $\mathrm{g}$ progesterone plus $2 \mathrm{mg}$ of estradiol benzoate i.m. (EB). On day 8, the DIV was removed and 500 ig cloprostenol was injected i.m., followed by administration of $1 \mathrm{mg}$ of EB i.m. $24 \mathrm{~h}$ later. Animals were assigned randomly to each of four treatments:T1) Early weaning at 10 days before the DIV insertion; T2) Early weaning the day of the DIV insertion; T3) Early weaning the day of withdrawal DIV and T4) No early weaning, calves were left with their respective mothers throughout the study period. All cows were fixed-time artificially inseminated $56 \mathrm{~h}$ after the DIV removal. In Exp. 1 and 2, follicular dynamics, time and distribution of the ovulation and pregnancy were evaluated by means of transrectal ultrasonography. Plasma progesterone concentrations were determined to evaluate the functionality and lifespan of the corpus luteum generated by the induced ovulation. At the time of the DIV removal, the dominant follicle diameter was larger $(p<0.05)$ in T2 $(11.2 \pm 2.5 \mathrm{~mm})$ than T3 $(7.3 \pm 2.0 \mathrm{~mm})$ in cows with low BCS (Exp. 1), but did not differ with $\mathrm{T} 1$ and T4 (10.3 \pm 2.4 and $8.2 \pm 2.8$, respectively). The T1 and T2 treatments presented a higher $(p<0.05)$ ovulation rate than T3 and T4 (77.8; 88.9; 40 and $40 \%$ respectively). Pregnancy rate was higher in T2 $(77.8 \%)$ than in the rest of treatments (T1= $55.6 \%, \mathrm{~T} 4=30 \%, \mathrm{~T} 4=20 \%$ ). In Exp. 2, no differences were found among treatments for all the evaluated parameters. In non-pregnant cows the corpus luteum had a normal lifespan, independently of treatment or BCS. In summary, early weaning performed 10 days before or the day of treatment start with progesterone/estrogen increased dominant follicle diameter (pre-ovulation) and ovulation rate in low BCS cows. Nevertheless, pregnancy rate was only improved when early weaning was done the day of the DIV insertion in the low BCS cows assigned to Exp. 1.

\section{INTRODUCCIÓN}

La progesterona o sus análogos (progestágenos) han demostrado su potenciali- dad para inducir celo-ovulación en ausencia de cuerpo lúteo en vacas con cría (Mapletoft et al., 1999; Yavas and Walton, 2000). Estos actúan induciendo una ovulación de fertilidad normal evitando la formación de un cuerpo lúteo de corta duración que ocurre después de la primera ovulación posparto. Es así que, la aplicación de progestágenos, minimiza la baja fertilidad del primer celo e induce sincronía de celoovulación facilitando la implementación de la inseminación artificial sistemática en rodeos de vacas y vaquillonas con funcionalidad ovárica deprimida (Mapletoft et al., 1999; Thompson et al., 1999).

Por otra parte, el amamantamiento o incluso la sola presencia del ternero al pie de la madre, es suficiente para prolongar el anestro posparto (Lamb et al., 1999). Este mecanismo fisiológico mediado por endorfinas y su interacción con los bajos niveles de estrógenos observados durante el posparto temprano, reduce la liberación pulsátil de LH y, consecuentemente, el desarrollo de un folículo ovulatorio (Short et al., 1990). La supresión temporal o definitiva de la lactancia, interrumpe este efecto negativo asociado al estado de anestro, mejorando las tasas de celo-ovulación (Mapletoft et al., 1999; Geary et al., 2001).

En trabajos realizados en Argentina, se ha demostrado que el destete precoz (60 días de edad aproximadamente) aplicado junto con progestágenos, son una excelente herramienta cuando los vientres se encuentran en condiciones nutricionales deficientes, como las que se presentan durante un período de sequía o en vacas primíparas, donde la lactancia deprime profundamente la actividad reproductiva. Asimismo, esta asociación ha demostrado una eficaz acción para aumentar la tasa de preñez y reducir el intervalo parto-concepción, cuando es aplicada en vacas cola de parición (Schiersmann et al., 1991; Scena et al., 1994), obteniéndose una mayor respuesta cuando este tipo de destete se realiza al finalizar un tratamiento para inducción de 


\section{DESTETE PRECOZEN VACAS TRATADAS CON PROGESTERONA}

celos con progestágenos (Bó et al., 2005).

El objetivo del presente trabajo fue generar información que explique los resultados biológicos que se obtienen al asociar el destete precoz con un tratamiento con progesterona intravaginal para la inducción/sincronización de celos-ovulación en vacas con cría, como también cuál o cuáles son las mejores combinaciones en función del momento de aplicación del destete.

\section{MATERIAL YMÉTODOS}

\section{ANIMALES Y EXPERIMENTOS}

Se realizaron dos experimentos, durante la primavera de 2007 (Experimento 1) y el otoño de 2008 (Experimento 2), con vacas multíparas de raza Hereford y Polled Hereford en la Estación Experimental Agropecuaria del INTA Concepción del Uruguay (provincia de Entre Ríos, Argentina; $32^{\circ} 29^{\prime}$ latitud Sur; $58^{\circ} 20^{\prime}$ longitud Oeste). En el Exp.1, se utilizaron 38 vacas con cría de $398,0 \pm 37,4 \mathrm{~kg}$ (promedio $\pm \mathrm{DE})$ de peso vivo, $3,3 \pm 0,8$ de condición corporal (CC, escala de 1 a 9; Herd and Sprott, 1986) y $55,7 \pm 8,8$ días posparto al inicio del tratamiento hormonal y en el Exp. 2, se utilizaron 35 vacas con cría de $507,1 \pm 43,6 \mathrm{~kg}$ de peso vivo, $5,4 \pm 0,9$ de CC y 57,5 $\pm 15,9$ días posparto al inicio del tratamiento hormonal. En función de la calificación de la CC de los animales, se definió a los experimentos $1 \mathrm{y}$ 2 como vacas de baja $C C$ y vacas de alta $C C$, respectivamente.

Durante el curso de los experimentos las vacas pastorearon sobre un pastizal natural, dominado por especies C4 (Andropogon lateralis, Axonopus affinis y Paspalum spp). Los terneros fueron destetados según las prácticas de manejo recomendadas para la utilización del alimento balanceado Ruter ${ }^{\circledR}$ (Asociación de Cooperativas Argentinas) y la metodología de Galli et al. (2005).

\section{DISEÑOEXPERIMENTAL YTRATAMIENTOS}

Las vacas de cada experimento fueron distribuidas según un diseño completamente aleatorizado a cuatro tratamientos: T1) destete precoz 10 días antes de colocar un dispositivo intravaginal (DIV) para bovinos impregnado con progesterona (DP-10); T2) destete precoz el día de colocación del DIV (DP0); T3) destete precoz el día del retiro delDIV (DP8); y T4) con cría (C/cría), los terneros permanecieron junto a sus madres. El número de vacas por tratamiento y la edad de los terneros al destete se presentan en la tabla I.

PROTOCOLO PARA INSEMINACIÓNARTIFICIAL A TIEMPOFIJO (IATF)

Las vacas fueron tratadas durante 8 días con un DIV para bovino (DIB, Syntex, Argentina) impregnado con $0,5 \mathrm{~g}$ de progesterona natural. Al momento de colocar los DIV (día 0) se aplicó una dosis de $2 \mathrm{mg}$ de benzoato de estradiol (BE; Benzoato de estradiol, Syntex, Argentina; i.m.). Al retiro de los DIV (día 8) se aplicó una dosis de 500 $\mu \mathrm{g}$ de cloprostenol sódico (Ciclase DL, Syntex, Argentina; i.m.) y 24 horas posretiro se aplicó $1 \mathrm{mg}$ de BE (Syntex, Argentina; i.m.). La inseminación artificial a tiempo fijo (IATF) fue realizada por un mismo operador a las 56 horas del retiro de los DIV con semen en pajuela congelado/descongelado de un toro Polled Hereford de fertilidad probada. En la figura 1 se presenta un esquema de los tratamientos realizados en función del protocolo de inducción/sincronización de celos utilizado.

\section{EVALUACIONES POR MEDIO DE ULTRASO- NOGRAFÍA}

Las evaluaciones ecográficas se realizaron por vía transrrectal con un ecógrafo de tiempo real (FALCOVET 100, Pie-Medical, Holanda) equipado con un transductor lineal de 6-8 MHz. El diámetro (mm) de los folículos fue calculado como el promedio de los diámetros vertical y transversal en su mayor sección. Se realizaron ecografías ováricas los días $-20 ;-10$ y 0 para caracterizar el estado ovárico previo al inicio del 


\section{VITTONE, ALLER, OTERO, SCENA, ALBERIO Y CANO}

tratamiento hormonal y se consideró como vaca cíclica a aquella que presentó un cuerpo lúteo (CL) en cualquiera de esos tres momentos. Al retirar los DIV (día 8), se observaron los ovarios de cada vaca y se consideró folículo dominante (FD) al folículo de mayor diámetro. Durante los días 9, 10 y 11 las ecografías se realizaron a intervalos de 12 horas. Como folículo ovulatorio (FO) se consideró al FD desde el día 9 hasta la ovulación. La ovulación se definió como la desaparición del FO seguido de la formación de un CL y el diámetro del FO al último registrado para esa estructura. El momento de la ovulación correspondió a la media en horas, entre la última observación del FO y la desaparición del mismo. La tasa de crecimiento (mm/día) del FO fue calculada como el promedio de las pendientes de regresión obtenidas para cada folículo. La formación y persistencia del CL fue observada durante los días 7, 12, 17 y 22 pos-IATF. El diagnóstico de gestación se realizó 31 días después de la IATF.

\section{Muestreo SANGUíNEO Y ANÁLISIS DE PROGESTERONA}

Las muestras de sangre fueron tomadas

Tabla I. Número de vacas por tratamiento (NVT)y edad (dias) al destete de los terneros (EDT) (media $\pm D E$ ). (Number of cows per treatment (NVT) and age (days) at weaning (EDT) (mean $\pm S D$ ) of calves).

\begin{tabular}{lcccc}
\hline & DP-10 & DP0 & DP8 & C/cría \\
\hline NVT & & & & \\
Exp. 1 & 9 & 9 & 10 & 10 \\
Exp. 2 & 9 & 9 & 8 & 9 \\
EDT & & & & \\
Exp. 1 & $45,1 \pm 10,6$ & $55,9 \pm 9,0$ & $64,1 \pm 7,9$ & - \\
Exp. 2 & $47,1 \pm 17,2$ & $56,8 \pm 15,8$ & $66,0 \pm 15,2$ & - \\
\hline
\end{tabular}

DP-10: destete 10 días antes de la colocación del DIV; DPO: destete el día de la colocación del DIV; DP8: destete el día del retiro del DIV y C/cría: sin destete. por punción de la vena yugular, colocadas en tubos con anticoagulante (EDTA) e inmediatamente centrifugadas a $2500 \mathrm{xg}$ durante 20 minutos ( $<1$ h desde la extracción). El plasma obtenido se conservó a $-20^{\circ} \mathrm{C}$ hasta el momento de realizar la determinaciones de progesterona. Se consideró en anestro a la vaca que presentó una concentración plasmática de progesterona por debajo de $1 \mathrm{ng} / \mathrm{ml}$ en los muestreos previos al inicio del protocolo de inducción/sincronización de celos (días $-20,-10$ y 0). Los días $7,12,17$ y 22 (pos-IATF) se tomaron muestras para evaluar la funcionalidad y la vida media del CL de todas las vacas que no resultaron preñadas, considerándose normales aquellas que mantuvieron niveles elevados de progesterona $(>1 \mathrm{ng} / \mathrm{ml})$ hasta el día 12 , disminuyendo posteriormente a niveles basales $(<1 \mathrm{ng} / \mathrm{ml})$. La determinación de progesterona plasmática se realizó mediante la prueba de radioinmunoensayo. Todas las muestras fueron analizadas por duplicado y el coeficiente de variación intraensayo fue $<9 \%$ para concentraciones comprendidas entre 0,1 y $40,0 \mathrm{ng} / \mathrm{ml}$. La sensibilidad del método utilizado fue de 0,1 $\mathrm{ng} / \mathrm{ml}$.

\section{ANÁLISIS ESTADÍSTICOS}

El diámetro del FD, el diámetro del FO y la tasa de crecimiento del FO fueron analizados mediante análisis de la varianza utilizando el procedimiento GLM (SAS, 1998). La concentración plasmática de progesterona se evaluó sólo en aquellos animales que ovularon y no resultaron preñados y fue analizada como medidas repetidas en el tiempo utilizando el procedimiento MIXED (SAS). Las tasas de ovulación (vacas que ovularon/vacas tratadas) y preñez (vacas preñadas/vacas tratadas) fueron analizadas mediante el procedimiento GENMOD (SAS) indicado para variables con distribución binomial. La distribución de las ovulaciones se comparó mediante el test exacto de Fisher. El momento de ovulación fue analizado mediante el test de Wilcoxon utilizando el 


\section{DESTETE PRECOZEN VACAS TRATADAS CON PROGESTERONA}

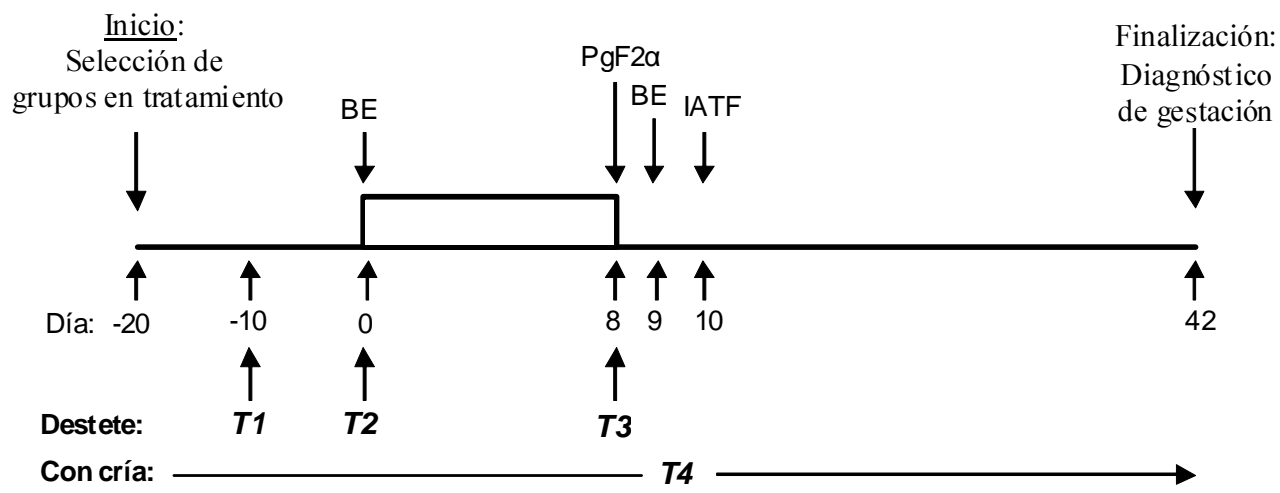

Figura 1. Presentación esquemática de los tratamientos. (Schematic presentation of the treatments).

procedimiento NPAR 1 WAY (SAS). El nivel de significancia estadística utilizado fue de 0,05. El modelo estadístico utilizado incluyó los efectos de la época de realización de los experimentos, el peso vivo inicial, la CC inicial y las posibles interacciones sobre las variables reproductivas en estudio.

\section{RESULTADOS}

\section{EXPERIMENTO1}

Dinámica folicular y características de la ovulación

Al iniciar el protocolo de inducción/ sincronización de celos, el 87\% (33/38) de las vacas se encontraba en anestro. El mayor diámetro del FD registrado correspondió al tratamiento DP0 difiriendo estadísticamente $(p<0,05)$ con el tratamiento DP8 y no observándose diferencias con DP-10 y $\mathrm{C} /$ cría. El diámetro del $\mathrm{FO}$ fue similar entre tratamientos, aunque se observó una tendencia $(\mathrm{p}=0,082)$ a presentar un mayor diámetro en el tratamiento DP0. La tasa de crecimiento promedio del FO desde el retiro del DIV hasta la ovulación, fue similar $(\mathrm{p}=$ 0,328 ) para todos los tratamientos. La mayor tasa de ovulación correspondió al tratamiento DP0, registrando diferencias $(\mathrm{p}=$ $0,041)$ con los tratamientos DP8 y C/cría. La distribución de las ovulaciones no difirió entre tratamientos $(p=0,431)$, observándose una mayor concentración hacia las 66 horas pos-retiro del DIV en los tratamientos DP8 y C/cría. En la tabla II se presentan los resultados de los parámetros ováricos analizados.

\section{Concentración de progesteronaplasmática en vacas no preñadas}

En las vacas que ovularon y no resultaron preñadas se registraron niveles crecientes de progesterona $(>1 \mathrm{ng} / \mathrm{ml})$ hasta el día 12 pos-IATF (figura 2), determinándose la ausencia de ciclos cortos en estos animales. Sin embargo, el bajo número $(n=6)$ de animales en esta condición imposibilitó la realización del análisis estadístico correspondiente. Adicionalmente, se realizó una determinación el día día 9 pos-retiro del DIV en las vacas que no ovularon $(\mathrm{n}=15)$, observándose a dos animales (DP8 $n=1$ y C/cría $n=1$ ) con niveles de progesterona mayores a 1 $\mathrm{ng} / \mathrm{ml}$. Estos dos casos fueron considerados como ovulaciones tardías ( $>72 \mathrm{~h}$ posretiro delDIV).

\section{Tasas de preñez y concepción}

Las tasas de preñez y concepción se presentan en la tabla III. El tratamiento DP0 registró la mayor tasa de preñez siendo esta diferencia significativa $(\mathrm{p}=0,043)$ sólo 


\section{VITTONE, ALLER, OTERO, SCENA, ALBERIO Y CANO}

Tabla II. Dinámica folicular y características de la ovulación en vacas de baja condición corporal tratadas con progesterona intravaginal y destete precoz realizado en diferentes momentos. (Follicular dynamics and ovulation characteristics in low body condition cows treated with intravaginal progesterone device and early weaning made at different times).

\begin{tabular}{lcccc}
\hline & DP-10 & DP0 & DP8 & C/cría \\
\hline Diámetro del FD al retiro del DIV (mm) & $10,3 \pm 2,4^{\text {ab }}$ & $11,2 \pm 2,5^{\mathrm{a}}$ & $7,3 \pm 2,0^{\mathrm{b}}$ & $8,2 \pm 2,8^{\mathrm{ab}}$ \\
Diámetro del FO (mm) & $11,9 \pm 0,8$ & $13,8 \pm 0,8$ & $10,1 \pm 1,1$ & $12,3 \pm 1,1$ \\
Tasa de crecimiento del FO (mm/día) & $1,1 \pm 0,2$ & $1,0 \pm 0,2$ & $1,7 \pm 0,3$ & $1,2 \pm 0,3$ \\
Tasa de ovulación (n) $^{\star}$ & $77,8(7 / 9)^{\mathrm{ab}}$ & $88,9(8 / 9)^{\mathrm{a}}$ & $40,0(4 / 10)^{\mathrm{b}}$ & $40,0(4 / 10)^{\mathrm{b}}$ \\
Momento de la ovulación (rango en horas) & $30-66$ & $42-66$ & $54-66$ & $66-66$ \\
\hline
\end{tabular}

${ }^{a b}$ En la misma fila, letras distintas: $p<0,05$. *vacas que ovularon/vacas tratadas. FD: folículo dominante. FO: folículo ovulatorio. DP-10: destete 10 días antes de la colocación del DIV; DP0: destete el día de la colocación del DIV; DP8: destete el día del retiro del DIV y C/cría: sin destete.

con los tratamientos DP8 y C/cría. La tasa de concepción no mostró diferencias $(\mathrm{p}=0,581)$ entre tratamientos por efecto del momento de aplicación del destete.

\section{EXPERIMENTO2}

\section{Dinámica folicular y características de la ovulación}

Al iniciar el protocolo de inducción/ sincronización de celos, el 60\% (21/35) de las vacas se encontraba ciclando. En este experimento, el diámetro promedio del FD al retiro del DIV, el diámetro promedio del FO y la tasa promedio de crecimiento de los folículos desde el retiro del DIV hasta la ovulación, no presentaron diferencias $(\mathrm{p}>0,05)$ entre tratamientos. La tasa de ovulación, el momento de ovulación y la distribución de las ovulaciones tampoco registraron diferencias $(p>0,05)$. La tasa promedio de ovulación de todos los tratamientos de este experimento fue del $80 \%$ y las ovulaciones tendieron a concentrase hacia las 66 horas pos-retiro del DIV. En la tabla IV se presentan los resultados de los parámetros ováricos analizados.

\section{Concentración plasmática de progesterona en vacas no preñadas}

La concentración plasmática de progesterona en las vacas que ovularon y no se preñaron, no difirió $(\mathrm{p}=0,618)$ entre tratamientos por efecto del momento de aplicación del destete. Por otro lado, se hallaron diferencias $(p<0,05)$ entre los momentos en que se ejecutaron los muestreos, presentando niveles crecientes de la hormona hasta el día 12 posterior al retiro del DIV. La concentración plasmática mayor a $1 \mathrm{ng} / \mathrm{ml}$ en el día 12 posterior al retiro del DIV en la totalidad de las vacas, indicó la ausencia de animales con ciclos cortos. En este experimento, también se determinó la concentración de progesterona de los 6 animales (DP$10 n=1$, DP0 $n=2$, DP $8 n=2$ y C/cría $n=1$ ) considerados como sin ovulación en las observaciones ecográficas y se detectó la ocurrencia de ovulaciones tardías $(>72 \mathrm{~h}$ pos-retiro del DIV) (nivel de progesterona $>1 \mathrm{ng} / \mathrm{ml}$ ). En la figura 3 se grafica la evolución de la concentración plasmática media de progesterona de todas las vacas que no resultaron preñadas.

\section{Tasas de preñez y concepción}

Las tasas de preñez y concepción se presentan en la tabla $\mathbf{V}$. No se encontraron diferencias $(p=0,366)$ en la tasa de preñez entre tratamientos. En este caso la tasa de concepción no pudo ser analizada estadísticamente debido al bajo número de animales y al resultado obtenido en el trata- 


\section{DESTETE PRECOZEN VACAS TRATADAS CON PROGESTERONA}

miento DP8 (100\% de preñez).

\section{DISCUSIÓN}

\section{DINÁMICAFOLICULARYCARACTERÍSTICASDE LA OVULACIÓN}

Al momento de retirar los DIV, el diámetro promedio del FD presentó diferencias entre tratamientos en las vacas de baja CC y sólo en dos tratamientos se observó un FD potencialmente ovulatorio (DP-10= $10,3 \pm 2,4$ y DP $0=11,2 \pm 2,5 \mathrm{~mm})$. Por el contrario, en los tratamientos DP8 y C/cría, el diámetro del FD no superó los $9 \mathrm{~mm}$, por lo que la probabilidad de continuar su desarrollo podría verse afectada por ser menos sensible a la LH (Ferraz et al., 2009). Los tratamientos que presentaron un $\mathrm{FD}>10 \mathrm{~mm}$ lograron mayores tasas de ovulación (DP-

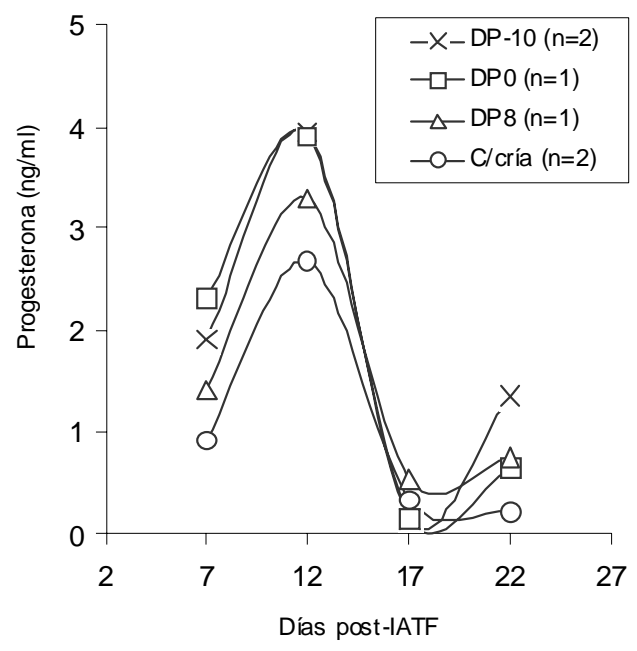

Figura 2. Concentración plasmática de progesterona en vacas de baja condición corporal (vacas que ovularon y no resultaron preñadas) tratadas con progesterona intravaginal y destete precoz realizado en diferentes momentos. (Plasma progesterone concentration in low body condition cows (ovulated and non-pregnancy cows) treated with intravaginal progesterone device and early weaning made at different times).
10 y DP0) respecto de aquellos con un folículo $<10 \mathrm{~mm}$, donde sólo ovuló el $40 \%$ de los animales (DP8 y C/cría). Estos resultados son consistentes con lo observado por Lucy et al. (1991), quienes establecieron que, folículos con un tamaño entre 10 y 15 $\mathrm{mm}$ son considerados dominantes y con la suficiente cantidad de receptores de LH para continuar su crecimiento y alcanzar la ovulación. El mayor tamaño del FD observado en el tratamiento DP0 en vacas de baja $C C$ podría haber sido una consecuencia de la combinación del efecto de la supresión del amamantamiento sobre la liberación de LH y el tratamiento con progesterona, ya que todos los grupos evaluados fueron tratados con el DIV y éstos también promueven el desarrollo folicular a través del incremento en la frecuencia de pulsos de LH una vez retirados (Roberson et al., 1989). Generalmente las vacas que presentan una pobre condición corporal durante la lactancia se encuentran en balance energético negativo, donde la frecuencia de pulsos de LH disminuye, afectando el tamaño final del FD (Roche et al., 2000). La mejora en el balance nutricional por efecto del destete podría ser otra de las razones que explique el mayor tamaño folicular observado en el tratamiento con destete al inicio (DP0) en vacas de baja $C C$.

Cuando el destete precoz fue aplicado a vacas de alta CC, el tamaño del FD fue mayor a $9 \mathrm{~mm}$ en todos los casos. La supresión de la lactancia no ejerció efecto sobre el tamaño del FD al finalizar el tratamiento con progesterona.

En el presente trabajo, en ambos experimentos, no se encontraron diferencias significativas entre tratamientos en el diámetro del FO, aunque el diámetro promedio de todos los folículos que alcanzaron la ovulación, fue algo superior en las vacas de alta $C C$ que en las vacas de baja condición $C C$ (Exp. $2=13,0 \pm 1,7 \mathrm{~mm}$ y Exp. $1=12,3 \pm 2,5$, respectivamente). La tasa de crecimiento folicular no presentó diferencias por efecto del amamantamiento en ambos experimen- 


\section{VITTONE, ALLER, OTERO, SCENA, ALBERIO Y CANO}

Tabla III. Tasas de preñez (TP) y concepción (TC) en vacas de baja condición corporal tratadas con progesterona intravaginal y destete precoz realizado en diferentes momentos. (Pregnancy (TP) and conception (TC) rates in low body condition cows treated with intravaginal progesterone device and early weaning made at different times).

\begin{tabular}{lccccc}
\hline & & DP-10 & DP0 & DP8 & C/cría \\
\hline TP & $\%$ & $55,6^{\text {ab }}$ & $77,8^{\mathrm{a}}$ & $30,0^{\mathrm{b}}$ & $20,0^{\mathrm{b}}$ \\
TP & $\mathrm{n}$ & $5 / 9$ & $7 / 9$ & $3 / 10^{\mathrm{b}}$ & $2 / 10^{\mathrm{b}}$ \\
TC & $\%$ & 71,4 & 87,5 & 75,0 & 50,0 \\
TC & $\mathrm{n}$ & $5 / 7$ & $7 / 8$ & $3 / 4$ & $2 / 4$ \\
\hline
\end{tabular}

${ }^{a b}$ En la misma fila, letras distintas: $p<0,05$. TP: vacas preñadas/vacas tratadas. TC: vacas preñadas/vacas que ovularon. DP-10: destete 10 días antes de la colocación del DIV; DP0: destete el día de la colocación del DIV; DP8: destete el día del retiro del DIV y C/cría: sin destete.

tos y concuerda con lo obtenido por Henao et al. (2000).

Generalmente, la tasa de ovulación obtenida por la aplicación de tratamientos con progesterona es aproximadamente $90 \%$ (Bó, 2005). Sin embargo, la respuesta a estos tratamientos está estrechamente ligada a la condición corporal de los vientres y a la presencia o ausencia del ternero al pie de la madre. Una restricción nutricional suficiente para reducir la condición corporal de los vientres, disminuye las concentraciones plasmáticas de LH (Richards et al., 1989) y el amamantamiento profundiza esta disminución (Williams et al., 1993), no siendo suficiente el aporte exógeno de progesterona para inducir la ovulación. El destete de los terneros durante las primeras 3 a 5 semanas posparto, incrementa la concentración plasmática de LH dentro de las 48 hs (Williams et al., 1993), presentándose un menor intervalo destete-ovulación en vacas de pobre condición corporal (Wettemann et al., 2003). En el presente estudio, en vacas de baja CC la mayor tasa de ovulación $(88,9 \%)$ se obtuvo cuando se realizó el destete al momento de iniciar el tratamiento con progesterona (DP0). En este caso, la asociación de progesterona y el destete ejerció un efecto positivo sobre la tasa de ovulación. En cambio, cuando se realizó el destete 10 días antes del tratamiento hormonal (DP-10), la tasa ovulatoria fue menor $(77,8 \%)$ y aún más baja $(40 \%)$ cuando se realizó al finalizar el tratamiento. Por lo cual, la respuesta no sólo está asociada a la utilización de estas prácticas combinadas, sino al momento en que éstas se combinan.

Las tasas de ovulación registradas en vacas de baja CC para los tratamientos DP8 y C/cría (40\%), estuvieron por debajo de lo informado (más del $60 \%$ ) por otros autores (Rhodes et al., 2002; Avilés et al., 2005). Es posible que, la presenciadel ternero en ambos tratamientos, sumado a la pobre CC de los vientres haya afectado negativamente la tasa de ovulación. En el caso de vacas de alta $C C$, no se obtuvo respuesta al destete en ninguno de los momentos en que fue aplicado. Estos resultados son similares a los obtenidos en otros trabajos en respuesta al uso de progesterona/estrógenos en vacas de óptima CC y cíclicas (75\% de ovulación), en las cuales ningún método de control del amamantamiento fue aplicado (Sorarrain et al., 2006). Según Bishop et al. (1994), la condición corporal está correlacionada con la frecuencia de pulsos de LH luego de realizar el destete precoz; y el incremento de las reservas de energía aumenta el estímulo pulsátil de LH que inicia la actividad ovárica. Por esta razón las vacas de baja CC presentaron una mayor respuesta al destete, ya que éste mejora su balance energético.

El momento de ocurrencia de las ovulaciones fue similar para todos los tratamientos, presentándose, en vacas de baja CC, a las $54 \mathrm{~h}$ posretiro del DIV en los tratamientos DP-10 y DP0 y a las 66 hs en los tratamientos DP8 y C/cría. En vacas de alta CC se observó un comportamiento similar en 


\section{DESTETE PRECOZ EN VACAS TRATADAS CON PROGESTERONA}

Tabla IV. Dinámica folicular y características de la ovulación en vacas de alta condición corporal tratadas con progesterona intravaginal y destete precoz realizado en diferentes momentos. (Follicular dynamics and ovulation characteristics in high body condition cows treated with intravaginal progesterone device and early weaning made at different times).

\begin{tabular}{lcccc}
\hline & DP-10 & DP0 & DP8 & C/cría \\
\hline Diámetro del FD al retiro del DIV (mm) & $10,2 \pm 0,7$ & $11,0 \pm 0,7$ & $9,2 \pm 0,7$ & $10,2 \pm 0,7$ \\
Diámetro del FO (mm) & $13,3 \pm 0,6$ & $13,3 \pm 0,6$ & $12,3 \pm 0,8$ & $13,1 \pm 0,6$ \\
Tasa de crecimiento del FO (mm/día) & $1,5 \pm 0,2$ & $1,0 \pm 0,2$ & $1,2 \pm 0,3$ & $1,2 \pm 0,2$ \\
Tasa de ovulación (n) & $88,9(8 / 9)$ & $77,8(7 / 9)$ & $62,5(5 / 8)$ & $88,9(8 / 9)$ \\
Momento de la ovulación (rango en horas) & $54-66$ & $42-66$ & $66-66$ & $42-66$
\end{tabular}

*vacas que ovularon/vacas tratadas. FD: folículo dominante. FO: folículo ovulatorio. DP-10= destete 10 días antes de la colocación del DIV; DP0= destete el día de la colocación del DIV; DP8= destete el día del retiro del DIV y $\mathrm{C} /$ cría $=$ sin destete.

todos los tratamientos (66 h). Varios autores (Cutaia et al., 2001; Ross et al., 2004) encontraron que, más del $80 \%$ de las ovulaciones se producen dentro de las $72 \mathrm{~h}$ posretiro de los DIV.

\section{CONCENTRACIÓNDE PROGESTERONAPLAS- MÁTICA EN VIENTRESNO PREÑADOS}

Los DIV con progesterona utilizados en tratamientos para inducir la ovulación, conducen a la formación de un cuerpo lúteo de vida media normal luego de su aplicación (Fike et al., 1997). En el presente trabajo, tanto en las vacas de baja CC como en las vacas de alta $C C$, no se observaron cuerpos lúteos con vida media corta, relacionándose este hecho al uso de progesterona y no al destete precoz, ya que, no se observaron diferencias entre tratamientos por efecto de la aplicación del destete.

\section{TASASDE PREÑEZY CONCEPCIÓN}

En las vacas de baja $C C$, las mayores tasas de preñez correspondieron a los tratamientos en los que se realizó el destete 10 días antes o al momento de colocar los DIV (DP-10 y DP0, respectivamente).

Los bajos porcentajes de preñez registrados en vacas de baja $C C$, cuando se realizó el destete precoz al retiro de los DIV y cuando las vacas mantuvieron la cría al pie, eran esperables, debido a que la tasa de ovulación fue del $40 \%$ para ambos tratamientos. El estado de anestro asociado a la baja condición corporal de los vientres y a la presencia del ternero hasta finalizar el tratamiento con progesterona, probablemente influyó sobre esta respuesta, ya que

Tabla $\boldsymbol{V}$. Tasas de preñez (TP) y concepción (TC) en vacas de alta condición corporal tratadas con progesterona intravaginal y destete precoz realizado en diferentes momentos. (Pregnancy (TP) and conception (TC) rates in high body condition cows treated with intravaginal progesterone device and early weaning made at different times).

\begin{tabular}{cccccc}
\hline & & DP-10 & DP0 & DP8 & C/cría \\
\hline TP & $\%$ & 44,4 & 33,3 & 62,5 & 22,2 \\
TP & $n$ & $4 / 9$ & $3 / 9$ & $5 / 8$ & $2 / 9$ \\
TC & $\%$ & 50,0 & 42,8 & 100,0 & 25,0 \\
TC & $n$ & $4 / 8$ & $3 / 7$ & $5 / 5$ & $2 / 8$ \\
\hline
\end{tabular}

TP: vacas preñadas/vacas tratadas. TC: vacas preñadas/vacas que ovularon. DP-10= destete 10 días antes de la colocación del dispositivo intravaginal; $\mathrm{DPO}=$ destete el día de la colocación del dispositivo intravaginal; DP8=destete el día del retiro del dispositivo intravaginal y $\mathrm{C} / \mathrm{crí} a=\sin$ destete. 
la presentación de ciclos estrales inducidos hormonalmente y las tasas de preñez resultantes, son dependientes del estado corporal de los vientres (Bastos et al., 2004) y la lactancia influye sobre este último.

En las vacas de alta $C C$ no se registraron diferencias entre tratamientos. Bó et al. (2001) observaron que, el efecto combinado del destete precoz y un tratamiento con progestágenos produjo un alto porcentaje de preñez $(63,9 \%)$ posterior a la IATF, realizando el destete 10 días antes del inicio del protocolo de sincronización en un rodeo con el $75 \%$ de vacas ciclando. Por otro lado, Bastos et al. (2003), informaron un $72,2 \%$ de preñez en vacas de buen estado corporal, al realizar el destete precoz al finalizar un tratamiento con progestágenos. Los resultados obtenidos por estos autores, fueron

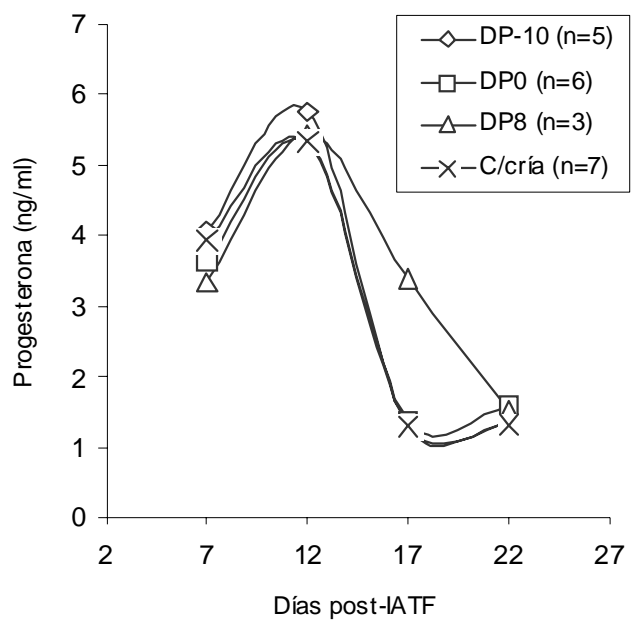

Figura 3. Concentración plasmática de progesterona en vacas de alta condición corporal (vacas que ovularon y no resultaron preñadas) tratadas con progesterona intravaginal y destete precoz realizado en diferentes momentos. (Plasma progesterone concentration in high body condition cows (ovulated and non-pregnancy cows) treated with intravaginal progesterone device and early weaning made at different times). superiores a los observados en el presente trabajo cuando se aplicó el destete en iguales momentos (DP-10 y DP8; 44,4 y 62,5\%, respectivamente). Sin embargo, es importante notar que, las tasas de preñez registradas para el grupo de vacas de alta $C C$ con destete al inicio del tratamiento con progesterona y en vacas sin destetar, no eran esperables, dado que, estos tratamientos presentaron un 77,8 y $88,9 \%$ de ovulación respectivamente.

En síntesis, el destete precoz ejerció sólo un efecto positivo sobre la tasa de preñez en vacas de baja $C C$, logrando el mayor impacto cuando se realizó al inicio del tratamiento con progestágenos.

\section{CONCLUSIONES}

En vacas de baja condición corporal, el destete precoz realizado 10 días antes o el día de inicio de un tratamiento con progesterona intravaginal, produce un aumento del tamaño del folículo dominante (preovulatorio) y una mayor tasa de ovulación que el destete realizado al finalizar el tratamiento o el control (sin destete). En vacas de alta condición corporal, no hay efecto del destete sobre los parámetros ováricos estudiados ni sobre la fertilidad. La vida media del cuerpo lúteo de las vacas no gestantes es normal, independientemente del tratamiento aplicado y de la condición corporal. La tasa de preñez sólo mejora cuando el destete precoz se aplica al comenzar el tratamiento con progesterona en vacas de baja condición corporal. Sin embargo, la coincidencia de un mayor tamaño del folículo dominante en las vacas destetadas 10 días antes o al iniciar el tratamiento hormonal, indicaría que, esos dos momentos serían los recomendados para ejecutar el destete y obtener una mayor probabilidad de éxito.

\section{AGRADECIMIENTOS}

Este trabajo se realizó gracias al aporte financiero otorgado por el Convenio de Vin- 


\section{DESTETE PRECOZEN VACAS TRATADAS CON PROGESTERONA}

culación Tecnológica firmado entre el Instituto Nacional de Tecnología Agropecuaria (INTA, Argentina) y la Asociación de Cooperativas Argentinas (ACA). Se agradece la participación en este trabajo a los Ingenie-

\section{BIBLIOGRAFÍA}

Avilés, M., Cutaia, L., Peres, L.C., Pincinato, D., Maraña Peña, D., Balla, E., Videla Dorna, I. y Bó, G.A. 2005. Efecto de $0,5 \mathrm{~g}$ o $1 \mathrm{~g}$ de progesterona sobre el momento de inicio de onda y ovulación en vacas y vaquillonas tratadas con benzoato de estradiol. Resúmenes. 6 Simposio Internacional de Reproducción Animal. IRAC. Córdoba. Argentina. 383 pp.

Bastos, G.D.M., Gonçalves, P.B.D., Machado, M.S.M., Restle, J., Neves, J.P., De Olivera, J.F.C., Farias, A.M., Siqueira, L. and Faturí, C. 2003. Hormonal induction of ovulation and early weaning in postpartum fertility of homozygous and heterozygous beef cows for microsatellite BMS3004. Rev. Bras. Zootecn., 32: 1093-1103.

Bastos, G.D.M., Brenner, R.H., Willke, F.W., Neves, J.P., Oliveira, J.F.C., Bragança, J.F.M., Machado, S.A., Porciúncula, P.M. and Gonçalves, P.B.D. 2004. Hormonal induction of ovulation and artificial insemination in suckled beef cows under nutritional stress. Theriogenology, 62: 847-853.

Bishop, D.K., Wettemann, R.P. and Spicer, L.J. 1994. Body energy reserves influence the onset of luteal activity after early weaning of beef cows. J. Anim. Sci., 72: 2703-2708.

Bó, G.A., Cutaia, L. Alisio, L. and Tegli, J. 2001. Inseminación artificial a tiempo fijo, resincronización de celos y destete precoz en vacas Braford. Rev. Braford, 16: 52-58.

Bó, G.A. 2005. Programa de inseminación artificial a tiempo fijo (IATF) en bovinos. Jornada de Actualización en Reproducción Bovina. Mercedes. Corrientes. Argentina. pp. 3-17.

Bó, G.A., Cutaia, L., Chesta, P., Balla, E., Picinato, D., Peres, L., Maraña, D., Avilés, M., Menchaca, A., Veneranda, G. y P.S. Baruselli. 2005. Implementación de programas de inseminación artificial en rodeos de cría de la Argentina. 6o Simposio Internacional de Reproducción Animal. IRAC. Córdoba. Argentina. pp. 97-128.

Cutaia, L., Moreno, D., Villata, L. and Bó, G.A. 2001. ros I. Galli y A. Monje, por su apoyo como asesores científicos y a los Señores E. Maidana, F. González, F. Ojeda, A. Taborda y J. Aldaz, por su colaboración en el trabajo de campo.

Synchrony of ovulation in beef cows treated with progesterone vaginal devices and estradiol benzoate administered at device removal at 24 hours later. Theriogenology, 55: 244.

Ferraz, H.T., Olivera Filho, B.D., Gambarini, L., Viu, M.A.O., Lopes, D.T. and Sousa, A.P.F. 2009. LH Pre-ovulatory peak in synchronized Nellore breed (Bos taurus indicus) females. Arch. Zootec., 58: 757-760.

Fike, K.E., Day, M.L., Inskeep, E.K.,Kinder, J.E., Lewis, P.E.R.E., Short, R.E. and Hafs, H.D. 1997. Estrus and luteal function in suckled beef cows that were in anoestrus when treated with an intravaginal device containing progesterone with and without a subsequent injection of estradiol benzoate. J. Anim. Sci., 75: 2009-2115.

Galli, I.O., Monje, A.R., Vittone, J.S., Sampedro, D. y Busto, C. 2005. Destete precoz en cría vacuna. Manual para la toma de decisiones y ejecución de la técnica. INTA. Serie Manual de Cría Vacuna. Vol. 2. pp. 94.

Geary, T.W., Whitter, J.C., Hallford, D.M. and Mac Neil, M.D. 2001. Calf removal improves conception rates to the Ovsynch and Co-Synch protocols. J. Anim. Sci., 79: 1-4.

Henao, G., Olivera-Ángel, M. and MaldonadoEstrada, J.G. 2000. Follicular dynamics during postpartum anestrus and the first estrous cycle in suckled or non-suckled Brahman (Bos indicus) cows. Anim. Reprod. Sci., 63: 127-136.

Herd, D. and Sprott, L. 1986. Body condition, nutrition and reproduction of beef cow. Texas Agricultural Extension Service. Bulletin B-1526.

Lamb, G.C., Miller, B.L., Lynch, J.M., Thompson, K.E., Heldt, C.A. and Löest, C.A. 1999. Twice daily suckling but not milking with calf presence prolongs postpartum anovulation. J. Anim. Sci., 77: 2207-2218.

Lucy, M.C., Staples, C.R., Michel, F.M. and Thatcher, W.W. 1991. Effect of feeding calcium soaps to early postpartum dairy cows on plasma prostaglandin $\mathrm{F}_{2 \alpha}$ luteinizing hormone, and 


\section{VITTONE, ALLER, OTERO, SCENA, ALBERIO Y CANO}

follicular growth. J. Dairy Sci., 74: 483-489.

Mapletoft, R.J., Bó, G.A., Martínez, M., Colazo, M., Caccia, M. y Adams, G.P. 1999. Control del desarrollo folicular y su uso en programas de in-seminación artificial a tiempo fijo en ganado de carne. $3^{\circ}$ Simposio Internacional de Reproducción Animal. IRAC. Córdoba. Argentina. pp. 51-69.

Rhodes, F.M., Burke, C.R., Clark, B.A., Day, M.L. and Macmillan, K.L. 2002. Effect of treatment with progesterone and oestradiol benzoate on ovarian follicular turnover in postpartum anoestrus cows and cows which have resumed oestrus cycles. Anim. Reprod. Sci., 69: 139150.

Richards, M.W., Wettemann, R.P. and Schoenemann, H.M. 1989. Nutritional anestrus in beef cows: Body weigth change, body condition, luteinizing hormone in serum and ovarian activity. J. Anim. Sci., 67: 15-20.

Roberson, M.S., Wolfe, M.W., Stumpf, T.T., Kittok, R.J. and Kinder, J.E. 1989. Luteinizing hormone secretion and corpus luteum function in cows receiving two levels of progesterone. Biol. Reprod., 41: 997-1003.

Roche, J.F., Makey, D. and Diskin M.D. 2000. Reproductive management of postpartum cows. Anim. Reprod. Sci., 60: 703-712.

Ross, J.P., Aller, J.F., Callejas, S.S., Butler, H. and Alberio, R.H. 2004. Estradiol given 0 or $24 \mathrm{~h}$ after the end of a progestagen treatment in postpartum suckled beef cows. Theriogenology, 62: 265273.

SAS Institute Inc. 1998. SAS/STAT ${ }^{\circledR}$ User's Guide, Version 6. Vol. 2. $14^{\text {th }}$ ed. Cary, NC. SAS Institute Inc. pp 846.

Scena, C., Monje, A.R. y Carou, N.E. 1994. Efecto del destete precoz sobre vacas primíparas cola de parición. Rev. Arg. Prod. Anim., 14: 123.

Schiersman, G.C.S., Mihura, H., Callejas, S.S. y Alberio, R.H. 1991. Efecto de un destete definitivo antes del segundo servicio en primavera sobre el comportamiento reproductivo de vacas primíparas paridas en otoño. Rev. Arg. Prod. Anim., 11: 167-175.

Short, R.E., Bellows, R.A., Staigmiller, R.B., Berardinelli, J.G. and Custer, E.E. 1990. Physiological mechanisms controlling anestrus and infertility in postpartum beef cattle. J. Anim. Sci., 68: 799-816.

Sorarrain, N., Lares, S.V., Vaca, R.J.A., Baldo, A. y de la Sota, R.L. 2006. Eficacia de la utilización de benzoato de estradiol a las $0 \circ 24$ horas del retiro de un dispositivo intravaginal con progesterona para inducir la ovulación en vacas para carne. Rev. Arg. Prod. Anim., 26 (Supl. 1): 305-306.

Thompson, K.E., Stevenson, J.S., Lamb, G.C., Grieger, D.M. and Loest, C.A. 1999. Follicular, hormonal, and pregnancy reponses of early postpartum suckled beef cows to $\mathrm{GnRH}$, norgestomet, and prostaglandin $\mathrm{F}_{2 \alpha}$. J. Anim. Sci., 77: 1823-1832.

Wettemann, R.P., Lents, C.A., Ciccioli, N.H., White F.J. and Rubio, I. 2003. Nutritional and suckling mediated anovulation in beef cows. J. Anim. Sci., (E. Suppl. 2): 48-59.

Williams G.L., McVey, W.R. and Hunter, J.F. 1993. Mammary somatosensory pathways are not required for suckling-mediated inhibition of luteinizing hormone secretion and delay of ovulation in cows. Biol. Reprod., 49: 13281337.

Yavas, Y. and Walton, J.S. 2000. Postpartum acyclicity in suckled beef cows: A review. Theriogenology, 54: 25-55. 